TAO, Vol. 11, No. 2, 381-392, June 2000

\title{
Trigger Time Synchronization in Shallow Seismic Exploration
}

\author{
Robert Sun ${ }^{1, *}$
}

(Manuscript received 1 May 1999, in final form 8 November 1999)

\begin{abstract}
An algorithm to synchronize the trigger time in reflection shallow seismic data is developed. Trigger time delay and shot statics (hereafter together referred to as the shot distortion) are handled simultaneously. The approach is to estimate the shot distortion on each common shot gather (CSG) and then to eliminate the distortion by shifting all the traces in that CSG. Relative shot distortion between two consecutive CSG's is estimated by computing the average of the time shifts of maximum cross-correlations between the first reflections on the corresponding traces in the two CSG's. The CSG number 1 is used as the datum on which shot distortion is estimated using receivers nearby shot location 1 . The shot distortion on each CSG is then calculated by relative shot distortion compared to CSG 1 . Traces in all the CSG's are then shifted to eliminate the shot distortion, thus synchronizing the trigger times. Testing against field data shows that alignment in common receiver gathers can be greatly improved, therefore supporting the validity of this algorithm.
\end{abstract}

(Key words: Shallow seismic, Trigger time distortion, Cross-correlation, Common shot gather)

\section{INTRODUCTION}

In a common depth point (CDP) reflection shallow seismic exploration, waves from a shot are recorded as a common shot gather (CSG). The arrival time of each event is assumed to be the traveltime of the wave from shot to receiver. Zero time is assumed to be the time at which the seismic waves are emitted from the shot. The trigger at the shot location senses the wave emission and initiates zero recording time.

Traces in different CSG's are sorted into either CDP gathers or common offset gathers to process normal moveout/dip moveout, stacking, migration, etc. Static corrections are implemented to remove shot statics and receiver statics to achieve good alignment of the traces (Hileman et al., 1968; Taner et al., 1974). One assumption underlying the correctness of these

\footnotetext{
${ }^{1}$ Department of Earth Sciences, National Chengkung University, Tainan, Taiwan, ROC

${ }^{\star}$ Corresponding author address: Dr. Robert Sun, Department of Earth Sciences, National Chengkung University, 1 Tahsueh Rd., Tainan, 701, Taiwan, ROC; E-mail: sun@geotech.et.ncku.edu.tw
} 
procedures is that the trigger initiates zero recording time precisely at the moment of formation of the initial seismic waves. Accordingly, the difference between the wave emitting time and the initiation of zero recording time (hereafter referred to as the trigger time delay) is desired to be zero or within some tolerable limit, say, $0.25 \mathrm{~ms}$.

However, in high-resolution shallow seismic exploration, it is not uncommon for real data to carry trigger time delay that is far beyond tolerable limit. A 48-channel, 100-shot shallow seismic line with shot and receiver intervals of both $2 \mathrm{~m}$ and near offset of $60 \mathrm{~m}$, is take $\mathrm{n}$ as an example of this. Figure 1 is the CSG number 1 with shot at location 1 and receivers at locations 31 through 78 (offsets $60 \mathrm{~m}$ through $154 \mathrm{~m}$ ) before static correction. Fair alignment (nearly static-free) between traces implies that receiver statics in this CSG are minor. Figures $2 \mathrm{a}$ and $\mathrm{b}$ are common receiver gathers (CRG's) with receivers at locations 100 and 110 respectively, and shots at locations 23 through 70 and locations 33 through 80 respectively, sorted before static correction (again). It is clear that some of the traces in Fig. 2 have poor alignment (with severe static problem). For instance, traces from shot locations 58 through 60 in both Figs. $2 \mathrm{a}$ and $\mathrm{b}$ have significant early arrival times compared to the other traces. Locations 33 through 78 are common to the receivers of the CSG in Fig. 1 and to the shot locations of the CRG's in Fig. 2. If the poor alignment of shots 58 through 60 in the CRG's in Fig. 2 is due to the weathering-induced shot statics, then it would be expected that receiver statics of similar amount appear at receivers 58 through 60 (channels 28 through 30) of the CSG in Fig. 1. Little receiver statics on these traces reduces the possibility of shot statics in Fig. 2. Plainly, the static problem in Fig. 2 is due to some other factors. The sole possible reason is trigger time delay. Martin and Jack (1990) also reported trigger time delay in petroleum exploration.

The maximum trigger time delay observed in Fig. 2 (the delay of the first reflection on the traces from CSG 58) is about $5 \mathrm{~ms}$. In high-resolution shallow seismic data where the peak period of signal is about $10 \mathrm{~ms}$, such delay can be equivalent to $1 / 2$ of a period or $\pi$ phase shift

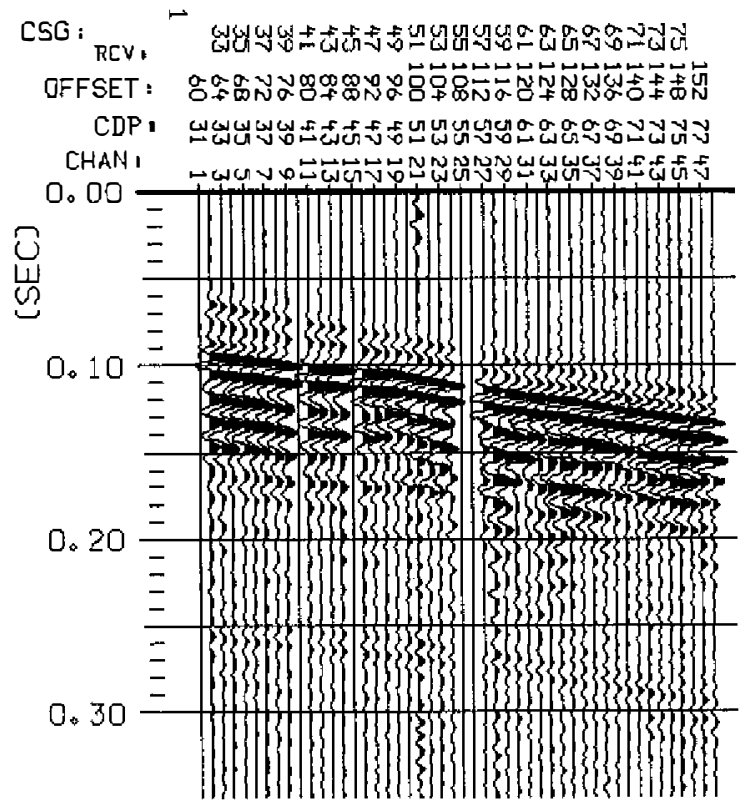

Fig. 1. Common shot gather (CSG) of shot 1 on a 100 -shot shallow seismic line. $\mathrm{RCV}=$ receiver; CHAN $=$ channel. 


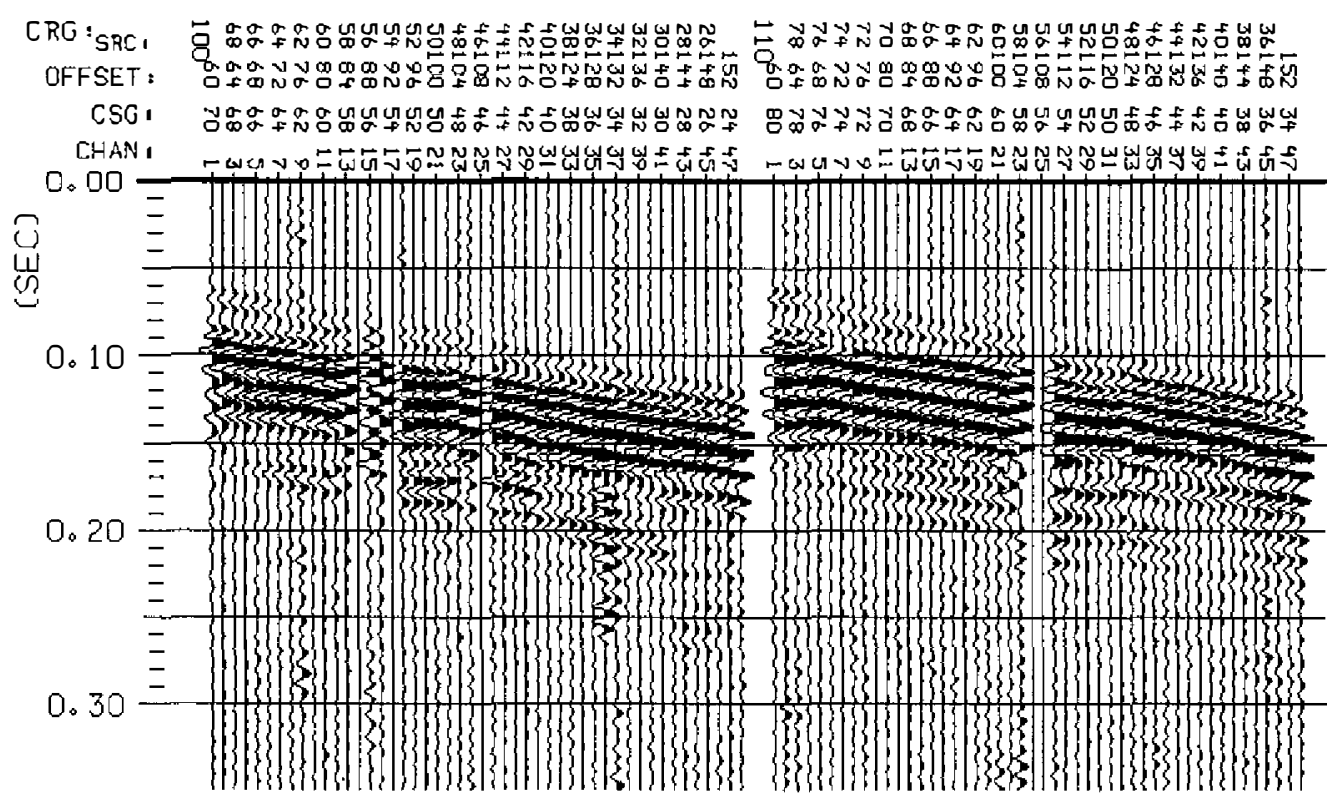

(a)

(b)

Fig. 2. Common receiver gathers (CRG's) with receivers at locations (a) 100 and (b) 110 on a 100 -shot shallow seismic line, sorted before residual static correction. $\mathrm{RCV}=$ receiver; $\mathrm{CHAN}=$ channel.

and can lead to unacceptable damage in stacking. Synchronizing trigger time is therefore a necessary procedure in shallow seismics.

In accordance with this situation, this paper develops an algorithm to synchronize trigger time. Traveltime is dependent on shot/receiver statics, velocity of the earth media (Taner et al., 1974) and trigger time delay. Separating each factor individually is difficult. It is desired to remove the shot/receiver statics and the rigger time delay. The strategy in this paper is first to perform residual statics correction (Yilmaz, 1987) as a priori procedure, to achieve good alignment between traces in a CSG. This will correct receiver statics and differential shot statics. That is, each CSG that is input to trigger time synchronization is assumed to have its first reflection arrival time concordant with the hyperbolic traveltime computed from the nearsurface velocity model. What remains to be corrected on a CSG is the trigger time delay and the part of shot statics that is common to all the traces in an entire CSG (the shot distortion, see (1) below). The approach in this paper is to quantify shot distortion on each CSG and to eliminate such distortion by shifting all the traces in that CSG. The task of trigger time synchronization is to achieve good alignment on all the CRG's.

The shallow seismic line where the data in Figs. 1 and 2 are acquired is used to illustrate the algorithm. This seismic line is in the southwestern Taiwan coastal plain. The weight-drop Elastic Wave Generator (EWG) manufactured by Bison Instruments, Inc., is used as the seismic source. $100-\mathrm{hz}$ vertical geophones are used as receivers. The sampling interval is $1 \mathrm{~ms}$ 
and the record length is $1023 \mathrm{~ms}$. The input data have undergone 60-10-160-10 hz (passes energy between $60 \mathrm{hz}$ and $160 \mathrm{hz}$ with $10 \mathrm{hz}$ ramps on both ends) bandpass filtering and badtrace elimination. Because the determination of shot distortion involves the only first reflection, all of the CSG and CRG figures in this paper are plotted only for time less than $350 \mathrm{~ms}$ which is more than enough to include first reflection.

\section{NEAR-SURFACE VELOCITY}

A near-surface velocity model is required in this algorithm and in the a priori residual static correction to compute the hyperbolic traveltime of the first reflection. In this research the near-surface velocity is estimated using NMO velocity analysis on the first reflection. That is, this algorithm does not attempt to deal with the velocity of the irregular weathered layer. Instead, this algorithm only seeks the RMS velocity that governs the arrival time of the first reflection, such that the first reflection has arrival time independent of the irregularity of the weathered layer.

\section{RELATIVE SHOT DISTORTION}

Relative shot distortion (difference of shot distortion) between two consecutive CSG's is found by comparing the arrival time difference of first reflection. A datum CSG is chosen on which the absolute trigger time delay is determined. The shot distortion on each CSG is then determined by the rigger time delay on the datum and the relative shot distortion compared to the datum.

Two consecutive CSG's on the seismic line, numbers 48 and 49 as in Figs. 3a and 3b respectively, are used to illustrate the determination of relative shot distortion. They are selected for illustration since there exists significant relative shot distortion between them, as can be observed on the CRG's in Fig. 2. The CSG's in Fig. 3 have been residual staticcorrected and are well aligned, as previously assumed. That is, the first reflections have arrival times concordant with the hyperbolic traveltime trajectory. This relation for CSG 48 (Fig. 3a) is presented in Fig. 4.

Let $M$ be the number of channels (here $M=48$ ). For channel i in CSG number $n$, let $t_{c}(i$, $n)$ and $t(i, n)$ be the hyperbolic traveltime and the arrival time of the first reflection, respectively. Good alignment in a CSG means that the difference between $t(i, n)$ and $t_{c}(i, n)$ is independent of channel number i:

$$
h(n)=t(i, n)-t_{c}(i, n) .
$$

If there is no shot distortion on CSG $n$, then the arrival time $t(i, n)$ should be equal to the traveltime $t_{c}(i, n)$ and we will have $h(n)=0$ in (1). But if there is in fact a shot distortion, then $h(n)$ represents the amount of shot distortion on CSG number $n$. It is evident that the relative shot distortion between CSG $\mathrm{n}-1$ and CSG $\mathrm{n}$ is the difference $\mathrm{k}(\mathrm{n})$ defined by

$$
k(n)=h(n)-h(n-1) .
$$




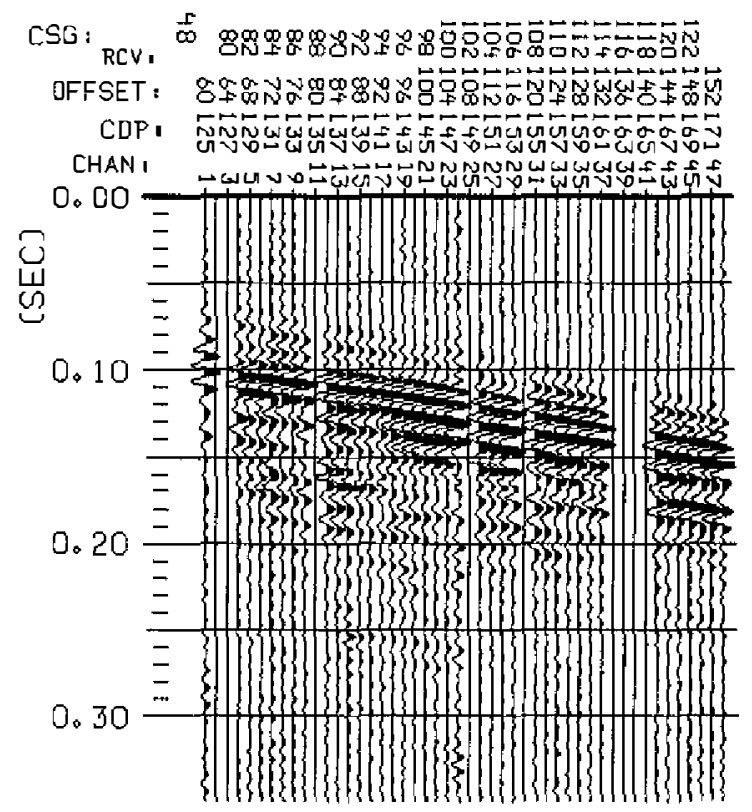

(a)

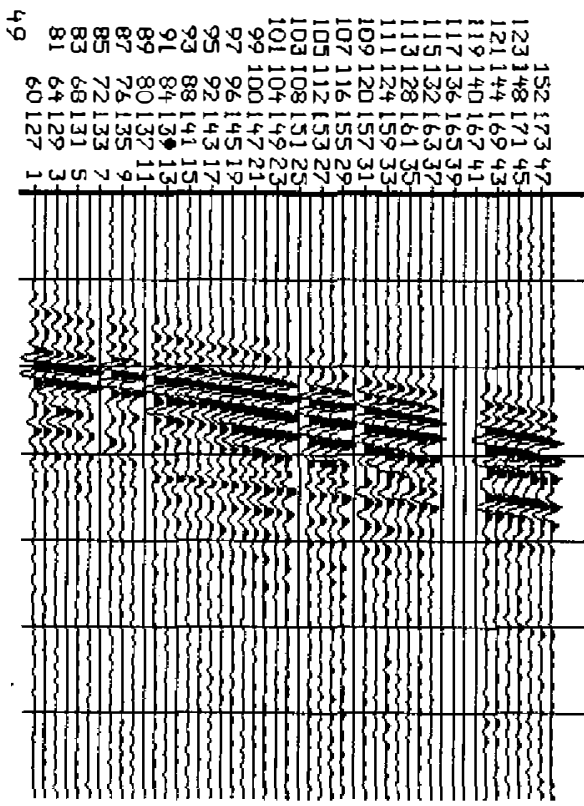

(b)

Fig. 3. Shallow seismic CSG's that are well aligned after residual static correction, (a) number 48 and (b) number 49 on the seismic line.

The NMO difference, differential shot statics and differential receiver statics are common to and implicitly included in both $t(i, n)$ and $t(i, n)$. It is eliminated at the subtraction in (1) and therefore does not appear in the shot distortion $\mathrm{h}(\mathrm{n})$ in (1) and in the relative shot distortion $\mathrm{k}(\mathrm{n})$ in (2).

Since the distortion $h(n)$ is uniform in the entire CSG as indicated in (1), ideally $h(n)$ can be determined using the first reflection on any single trace in that CSG, and, in the perfect case, any single $\mathrm{i}$ is sufficient to determine $\mathrm{k}(\mathrm{n})$ by [using (2)]:

$$
\mathrm{k}(\mathrm{n})=\left[\mathrm{t}(\mathrm{i}, \mathrm{n})-\mathrm{t}_{\mathrm{c}}(\mathrm{i}, \mathrm{n})\right]-\left[\mathrm{t}(\mathrm{i}, \mathrm{n}-1)-\mathrm{t}_{\mathrm{c}}(\mathrm{i}, \mathrm{n}-1)\right] .
$$

Consequently, ideally the cross-correlation between the first reflection on channel $i$ in CSG $\mathrm{n}-1$ and that in CSG $\mathrm{n}$ should have its maximum at time shift of $\mathrm{k}(\mathrm{n})$.

In computing the cross-correlation, the first reflection is windowed so as to separate it from other portions of a CSG. As the CSG is well aligned (after residual static correction), the windows can be chosen such that the upper and lower boundaries are concordant with the hyperbolic traveltime $t_{c}(i, n)$. That is, for channel number $i$, the beginning time $t_{1}(i, n)$ and the ending time $t_{2}(i, n)$ of the window are related to $t_{c}(i, n)$ by:

$$
\mathrm{t}_{1}(\mathrm{i}, \mathrm{n})=\mathrm{t}_{\mathrm{c}}(\mathrm{i}, \mathrm{n})-\Delta \mathrm{t}_{1},
$$

and

$$
\mathrm{t}_{2}(\mathrm{i}, \mathrm{n})=\mathrm{t}_{\mathrm{c}}(\mathrm{i}, \mathrm{n})+\Delta \mathrm{t}_{2},
$$




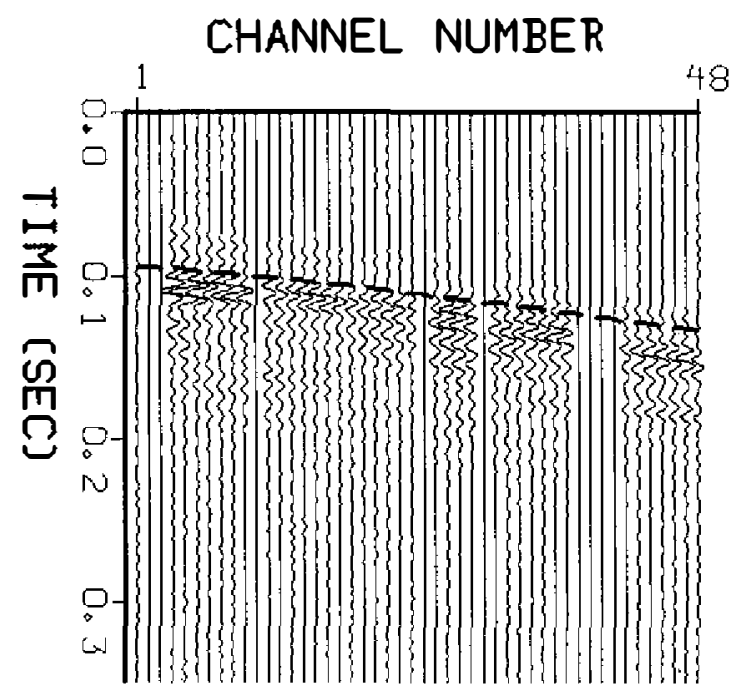

Fig. 4. In the well aligned CSG in Fig. $3 a$, the hyperbolic traveltime trajectory (dashed line) and the arrival time of the first reflection are well concordant.

for some positive time intervals $\Delta \mathrm{t}_{1}$ and $\Delta \mathrm{t}_{2}$. Here $\Delta \mathrm{t}_{1}$ and $\Delta \mathrm{t}_{2}$ should be selected so that the time interval from $t_{1}(i, n)$ to $t_{2}(i, n)$ encompasses the entire first reflection energy. Ramps are given on both ends of the window to avoid sharp amplitude change. For the CSG's in Fig. 3 with $\Delta \mathrm{t}_{1}=5 \mathrm{~ms}$ and $\Delta \mathrm{t}_{2}=30 \mathrm{~ms}$ chosen, the windowed first reflections are presented in Fig. 5.

Let $f_{0}(t, i, n)$ represent the function of the windowed first reflection on trace $i$ in CSG $n$, where $t$ is time. The onset of the first reflection on $f_{0}(t, i, n)$ should be at time $h(n)+t_{c}(i, n)$ as indicated by (1). If $f_{0}(t, i, n)$ is shifted by the amount of traveltime $t_{c}(i, n)$ to obtain the shifted function

$$
f(t, i, n)=f_{0}\left(t+t_{c}(i, n), i, n\right),
$$

then the onset of the first reflection on $f(t, i, n)$ should be at time $h(n)$, as can be understood by (1). Similarly, the shifted function

$$
f(t, i, n-1)=f_{0}\left(t+t_{c}(i, n-1), i, n-1\right)
$$

has the onset of the first reflection at time h(n - 1). Then the cross-correlation (Båth, 1979)

$$
\phi(\tau, \mathrm{i}, \mathrm{n})=\int_{-\infty}^{\infty} \mathrm{f}(\mathrm{t}, \mathrm{i}, \mathrm{n}-1) \mathrm{f}(\mathrm{t}+\tau, \mathrm{i}, \mathrm{n}) \mathrm{dt}
$$

reveals the 'extent of correlation' between the first reflections on the two functions $f(t, i, n-1$ 1) and $f(t, i, n)$. Let $\tau_{m}(i, n)$ represent the time shift of maximum cross-correlation:

$$
\phi\left(\tau_{\mathrm{m}}(\mathrm{i}, \mathrm{n}), \mathrm{i}, \mathrm{n}\right)=\max [\phi(\tau, \mathrm{i}, \mathrm{n})],
$$

then $\tau_{\mathrm{m}}(\mathrm{i}, \mathrm{n})$ indicates the relative shot distortion $\mathrm{k}(\mathrm{n})$ in (2) and (3), i.e.,

$$
\tau_{m}(i, n)=k(n)=h(n)-h(n-1) .
$$




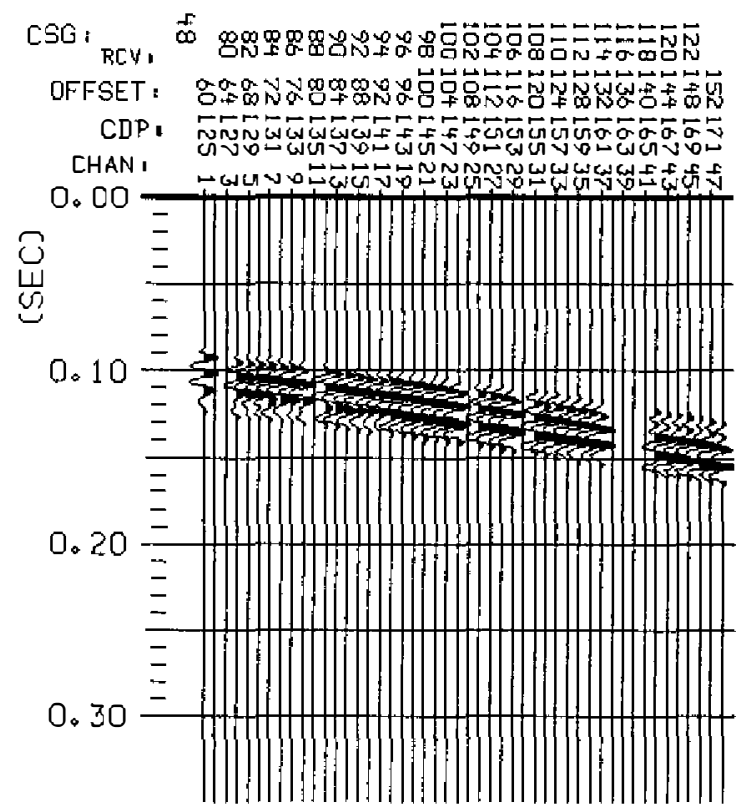

(a)

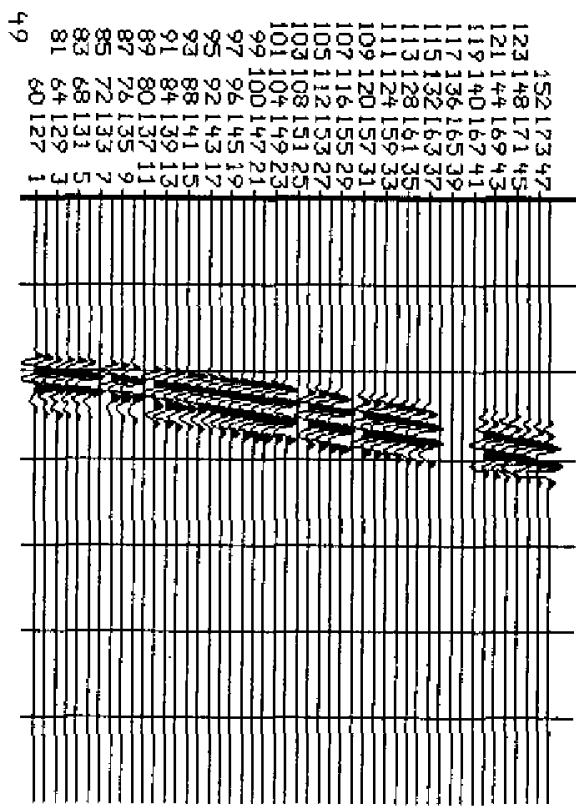

(b)

Fig. 5. Windowed first reflections of CSG's (a) 48 in Fig. 3a and (b) 49 in Fig. 3b.

As shot distortion $\mathrm{h}(\mathrm{n})$ is uniform over all the traces in a CSG, ideally any single $\mathrm{i}$ (where $\mathrm{l}<\mathrm{i}<\mathrm{M}$ ) is sufficient to determine $\mathrm{k}(\mathrm{n})$ using (8).

Using (6), there will be one cross-correlation curve for each $i$. For the first reflections in Fig. 5, there should be 48 cross-correlation curves as presented in Fig. 6. But curves of bad traces are eliminated and do not appear in Fig. 6. The maximum cross-correlation on each curve can be clearly observed, and the time shift $\tau_{m}(i, n)$ of the maximum cross-correlation is presented in Fig. 7.

If $\tau_{m}(i, n)$ were error-free, the time shift in Fig. 7 would be constant for all channels. Actually though, Figure 7 shows that $\tau_{m}(i, n)$ is nearly constant but does vary slightly from channel to channel. With a few exceptions, most of the $\tau_{\mathrm{m}}$ values are within $\pm 0.2 \mathrm{~ms}$ of the average. Those $\tau_{\mathrm{m}}$ far away from the average are possibly due to noise in the seismic data or malfunctioning electronic circuit in data acquisition and are taken as bad. To determine the single-valued relative shot distortion $k(n)$, the average over all the good $\tau_{m}(i, n)$ is calculated. Those bad $\tau_{m}$ are indicated by ' $x$ ' in Fig. 7 and are ignore in calculating the average. In formula, taking $\tau_{m}(i, n)=0$ if channel $i$ is bad, we have

$$
k(n)=\sum_{i=1}^{M} \tau_{m}(i, n) / M_{L},
$$

where $M_{L}$ is the number of good $\tau_{m}$. 


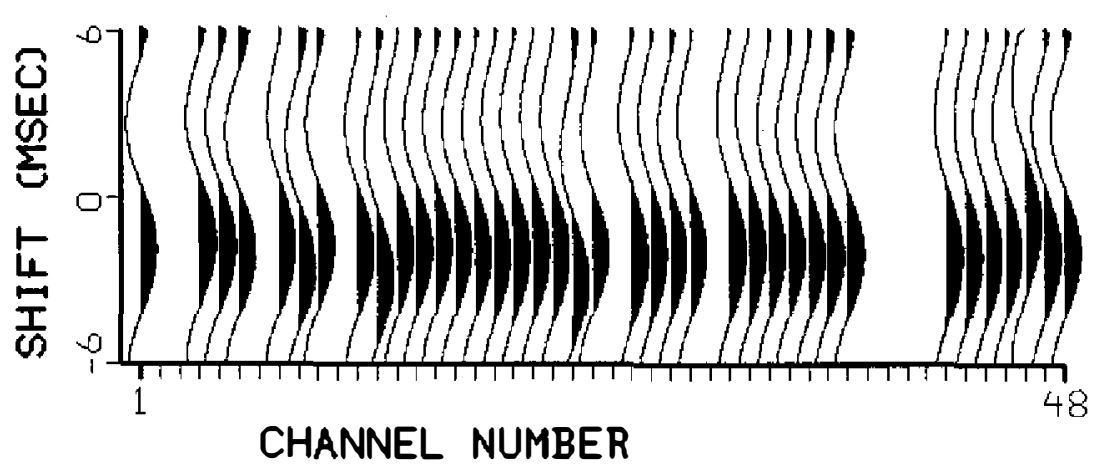

Fig. 6. Cross-correlations between corresponding races in the CSG's in Figs. 5a and $5 \mathrm{~b}$. Positive value is blackened and negative value is not.

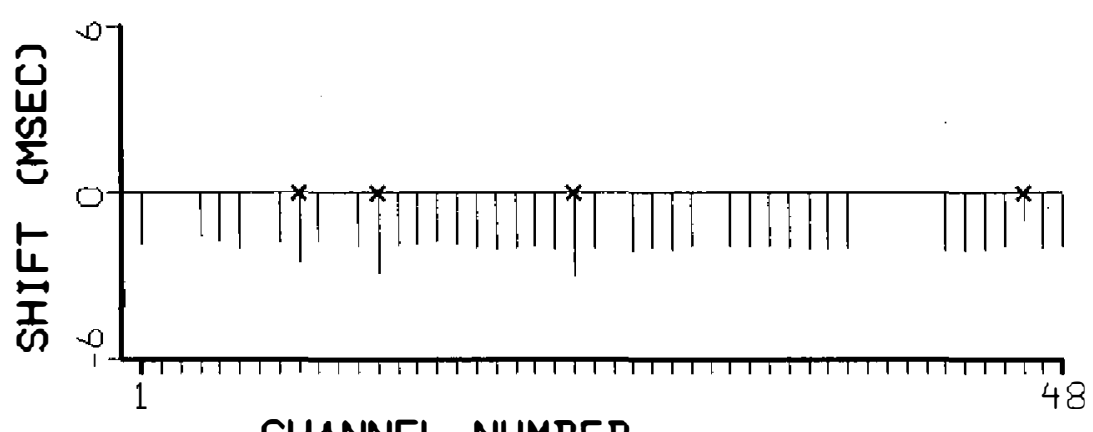

\section{CHANNEL NUMBER}

Fig. 7. Time shifts of the maximum cross-correlations in Fig. 6.

\section{THE DATUM}

The shot distortion on CSG $\mathrm{N}$ is represented by $\mathrm{h}(\mathrm{N})$ [eq. (1)]. If the shot distortion $\mathrm{h}(1)$ of CSG 1 can be determined, then the shot distortion on all the CSG's can be determined by accumulating the relative shot distortion between consecutive CSG's. In this paper the shot distortion on CSG 1 is determined by linear extrapolation of arrival time at receivers near location 1. Four geophones are spread near location 1 with $0.3 \mathrm{~m}$ to $1.2 \mathrm{~m}$ offsets and $0.3 \mathrm{~m}$ spacing. Seismic wave is emitted from location 1 and the wave recorded with 0.25 ms sampling interval is presented in Fig. 8a. The onset time of direct arrival is presented in Fig. 8b. Also presented in Fig. $8 \mathrm{~b}$ is a straight line that represents the onset time as a function of offset by least-squares modeling.

In Fig. 8 the maximum offset is only $1.2 \mathrm{~m}$, we can consider the travel time of the direct arrival to be a linear function of offset. Consequently the onset time of the wave at the shot location (location 1, zero offset) can be extrapolated by the least-squares modeling line in Fig. $8 \mathrm{~b}$ as $-0.2 \mathrm{~ms}$. This negative onset time at shot location 1 means that the trigger is delayed by this amount. This provides the shot distortion on CSG 1 to be $h(1)=0.2 \mathrm{~ms}$. 

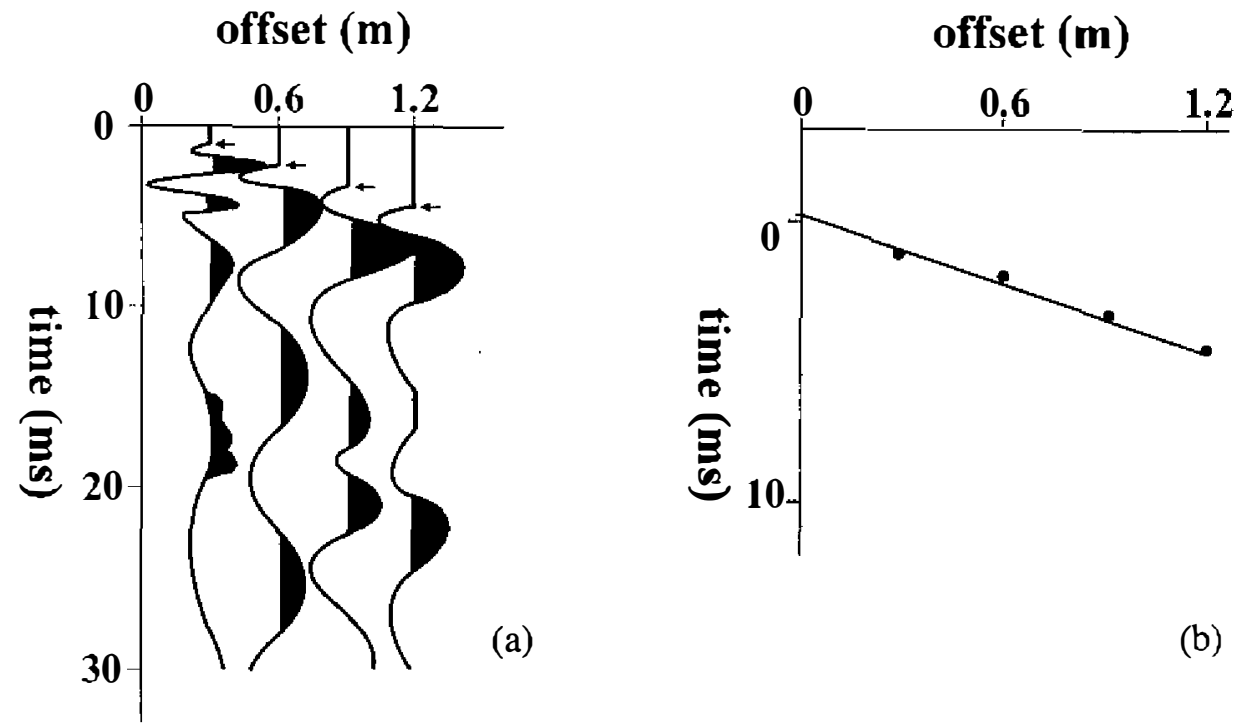

Fig. 8. Finding shot distortion on CSG 1, the datum. The shot is at location 1 and four receivers are at 0.3 to $1.2 \mathrm{~m}$ offset with $0.3 \mathrm{~m}$ spacing. (a) The shot record of $0.25 \mathrm{~ms}$ sampling interval and $30 \mathrm{~ms}$ record length. The onset of direct arrival on each trace is pointed by an arrow. (b) The onset time of the direct arrival with a straight line to model the onset time by least squares.

\section{SHOT DISTORTION}

The result in (9) reveals only the relative shot distortion between consecutive CSG's. Using (1), the shot distortion $h(N)$ for CSG $N$ (where $N \geq 2$ ) can be calculated by adding the shot difference $k(n)$, for $n$ of 2 to $N$, to $h(1)$ :

$$
h(N)=h(1)+\sum_{n=2}^{N} k(n) .
$$

As mentioned earlier, the shot distortion $\mathrm{h}(\mathrm{N})$ in (10) implicitly includes trigger time delay and part of the shot statics.

\section{TESTING SYNCHRONIZATION}

The algorithm in this paper is tested against the 100-shot seismic line from which the CSG in Fig. 1 and the CRG's in Fig. 2 come. The CSG data after residual static correction are the inputs to trigger time synchronization. The shot distortions calculated using (10) are presented in Fig. 9 for all the CSG's. Figure 9 shows that the distortion on CSG 58 is about -4.5 $\mathrm{ms}$, which means that the trigger time delay is $4.5 \mathrm{~ms}$ if the distortion is from the delay only. Some of the CSG's have positive distortion. Since trigger time delay is always positive that 
induces negative distortion, positive distortion is possibly from shot statics.

The traces in all the CSG's are shifted to eliminate the shot distortion using the information in Fig. 9, and the resulting synchronized data are presented with CRG numbers 100 and 110 (again) in Fig. 10. Comparing Fig. 10 with Fig. 2 which shows the CRG's before synchronization, the alignment in Fig. 10 is greatly improved. To illustrate, the severe trigger time delay on CSG's 58 through 60 are eliminated.

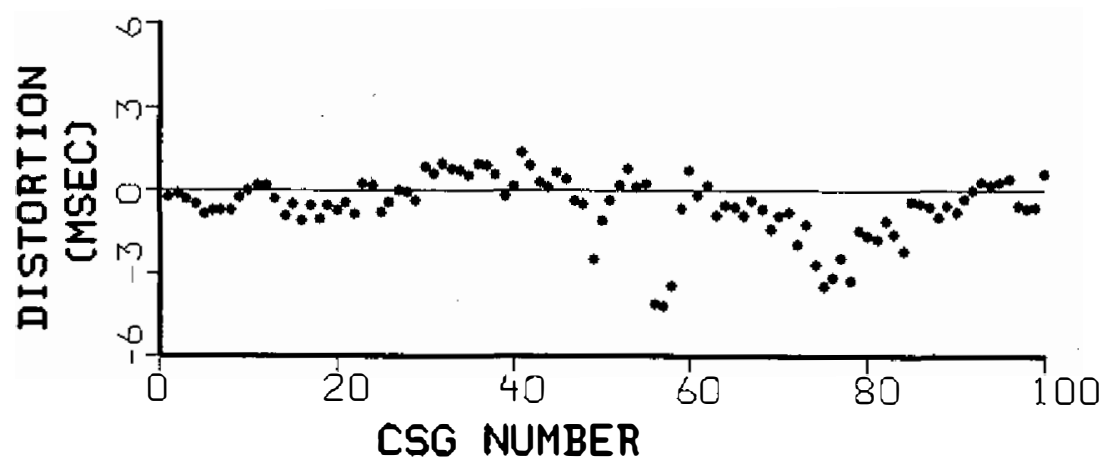

Fig. 9. Shot distortions on the CSG's calculated from (10).



(a)

(b)

Fig. 10. CRG's with receivers at locations (a) 100 and (b) 110 on the 100 -shot shallow seismic line, sorted after trigger time synchronization on the seismic line. $\mathrm{RCV}=$ receiver; $\mathrm{CHAN}=$ channel. 


\section{CONCLUSIONS AND DISCUSSION}

The maximum trigger time delay ever observed by the author is about $\pm 5 \mathrm{~ms}$. Martin and Jack (1990) have reported trigger time delay of up to $8 \mathrm{~ms}$. Such a delay might be negligible in petroleum prospecting where the peak period of the signal is $30 \mathrm{~ms}$ or more, but not in highresolution shallow seismic exploration.

The author has yet to find an answer as to why trigger time is distorted. It may be conjectured that the distortion is due to the mechanical uncertainty of the trigger. However, the testing data in this paper are generated by a weight-drop source, the Elastic Wave Generator, and each CSG is the stack of waves from at least 2 impacts. The source is at the earth's surface rather than in a shot hole, therefore no uphole time is involved. The trigger is installed on the edge of the impact plate. Figure 11 presents channel 10 of three shot records, each with a single impact, at (fixed) location 58 where trigger time delay is severe (see Fig. 2). Little arrival time difference can be observed in these traces (of the same shot and receiver) implies that little trigger time delay exists between impacts at the same shot location. Thus mechanical uncertainty is possibly not the origin. The non-linear property of the near-surface earth media (Martin and Jack 1990) might be the origin of trigger time delay. Moreover, it is worth noting that the CRG's in Fig. 2 show that shot locations with significant shot distortion are in fact grouped instead of being sparsely distributed.

The relative shot distortion between two consecutive CSG's is determined by finding the time shift of maximum cross-correlation. For such determination to be effective, the waves in the CSG's must be composed of similar wavelets. In other words, the same seismic source for data acquisition must be used in the entire seismic line, as in fact is usually the case.

Error always exists in seismic data, thus errors in cross-correlation [eq. (6)], in time shift of maximum cross-correlation [eq. (7)], in relative shot distortion [eq. (9)] and in shot distortion [eq. (10)] are inevitable. The validity of these estimations is based on the assumption that error is random. Such assumptions are generally satisfied.

The algorithm in this paper assumes that the near surface has velocity lower than the layers below, which is usually the case. But if the near surface has higher velocity than below, the validity of this algorithm will be reduced.

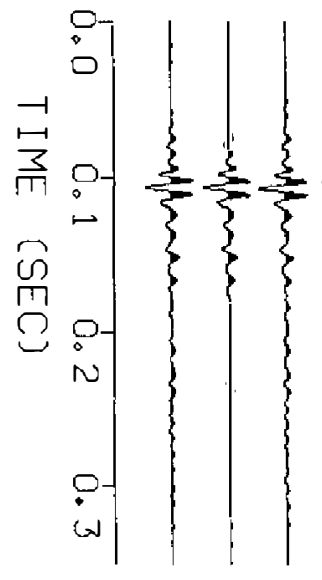

Fig. 11. Channel 10 of 3 shot records, each with single weight-drop impact, at (fixed) location 58. Left race: first shot; center trace: second shot; right trace: third shot. 
A CSG is necessary to serve as a datum to obtain the shot distortions on all the CSG's. In this paper the author choose CSG 1 as the datum by finding its trigger time delay. However, any CSG can be used as the datum. Moreover, multiple data can be used as well.

A good reflection is required for this algorithm to work properly. The reflector that generates this reflection can be either horizontal or dipping, as long as its travel time curve can be obtained. If this is not the case, this algorithm cannot be used.

Vertical component contains predominantly $\mathrm{P}$-wave reflections which is the main energy used in this paper. With aperture increases, $\mathrm{S}$-wave content in the vertical component will increase. However, S-waves have much lower velocity than $\mathrm{P}$-waves do and therefore have much longer arrival times and usually does not interfere the first P-wave reflection.

The task in this paper is to develop an algorithm to synchronize rigger time on the CSG's. Successfully correcting the shot distortion in the testing example gives proof of the effectiveness of this algorithm. However, amplitude and phase variations are not involved in this algorithm and will remain uncorrected.

Missing traces are somewhat frequent in reflection seismic data. The handling of missing traces is not explicitly addressed in this text, but the testing example includes quite a few missing races. The satisfactory results imply that the present algorithm handles missing traces properly as long as they are not too many, say not more than $30 \%$. But this algorithm may fail if a majority, say $50 \%$ or more, of the races are missing.

For a seismic line of $100 \mathrm{CSG}$ 's with 48 channels and $1024 \mathrm{~ms}$ record length, one execution of trigger time synchronization takes only about 40 seconds of CPU time on an HP715 workstation. Such a tiny CPU time is easily affordable.

Acknowledgments The research leading to this paper is funded by the National Science Council of the Republic of China on Taiwan under grant no. NSC86-2116-M-006-008. Thanks to Dr. C. H. Wang at National Central University in Taiwan for providing some of the important subprograms.

\section{REFERENCES}

Båth, M., 1979: Spectral Analysis in Geophysics, Elsevier, New York, p.77.

Hileman, J. A., P. Embree, and J. C. Pfleuger, 1968: Automated static corrections. Geophys. Prospecting, 16, 326-358.

Martin, J. E., and I. G. Jack, 1990: The behaviour of a seismic vibrator using different phase control methods and drive levels. First Break, 8, 404-414.

Taner, M. T., F. Koehler, and K. A. Alhilali, 1974: Estimation and correction of near-surface time anomalies. Geophysics, 41, 441-463.

Yilmaz O., 1987: Seismic Data Processing, Society of Exploration Geophysics, Tulsa, Oklahoma. 